\title{
POWER SERIES AND SMOOTH FUNCTIONS EQUIVALENT TO A POLYNOMIAL
}

\author{
WOJCIECH KUCHARZ
}

\begin{abstract}
An algebraic criterion is given for a power series in $n$ variables over a field of characteristic 0 to be equivalent to a polynomial in $n-k$ variables over the ring of power series in $k$ variables. For convergent power series over the reals or complexes a geometric interpretation of the criterion is established. An analogous sufficient condition is obtained for germs of smooth functions. Most of the previously known results follow easily from the criterion.
\end{abstract}

1. Introduction. Let $\mathbf{K}$ be a field of characteristic 0 . We denote by $F_{n}=$ $\mathbf{K}\left[\left[x_{1}, \ldots, x_{n}\right]\right]$ the ring of formal power series in $x_{1}, \ldots, x_{n}$ over $\mathbf{K}$. If $\mathbf{K}$ is equipped with an absolute value (in that case we always assume that $\mathbf{K}$ is complete), then $O_{n}=\mathbf{K}\left\{x_{1}, \ldots, x_{n}\right\}$ denotes the subring of $F_{n}$ of convergent power series. Let $A_{n}$ be either of the rings $F_{n}$ or $O_{n}$. Two power series $f$ and $g$ in $A_{n}$ are said to be equivalent if there exists a $\mathbf{K}$-automorphism of $A_{n}$ transforming $f$ onto $g$. Our goal in this note is to give criteria for a power series to be equivalent to a power series which is a polynomial with respect to at least some of the variables.

Given a $p$-tuple $\varphi=\left(\varphi_{1}, \ldots, \varphi_{p}\right)$ of elements of $A_{n}$, we denote by $I(\varphi)$ the ideal of $A_{n}$ generated by all $p \times p$ minors of the $p \times(p+n)$ matrix

$$
M(\varphi)=\left(\begin{array}{ccccccc}
\varphi_{1} & 0 & \ldots & 0 & \frac{\partial \varphi_{1}}{\partial x_{1}} & \ldots & \frac{\partial \varphi_{1}}{\partial x_{n}} \\
0 & \varphi_{2} & \ldots & 0 & \frac{\partial \varphi_{2}}{\partial x_{1}} & \ldots & \frac{\partial \varphi_{2}}{\partial x_{n}} \\
\vdots & \vdots & & \vdots & \vdots & & \vdots \\
0 & 0 & \cdots & \varphi_{p} & \frac{\partial \varphi_{p}}{\partial x_{1}} & \ldots & \frac{\partial \varphi_{p}}{\partial x_{n}}
\end{array}\right)
$$

Note that $I\left(u_{1} \varphi_{1}, \ldots, u_{p} \varphi_{p}\right)=I\left(\varphi_{1}, \ldots, \varphi_{p}\right)$ if $u_{1}, \ldots, u_{p}$ are units in $A_{n}$. It is well known that $A_{n}$ is a factorial ring and one easily shows that every element $f$ in the maximal ideal $m\left(A_{n}\right)$ of $A_{n}$ can be written as $f=\varepsilon f_{1}^{\lambda_{1}} \cdots f_{p}^{\lambda_{p}}$, where $\varepsilon$ belongs to $\mathbf{K}^{*}=\mathbf{K} \backslash\{0\}, f_{1}, \ldots, f_{p}$ are irreducible, relatively prime elements of $A_{n}$ and $\lambda_{1}, \ldots, \lambda_{p}$ are positive integers. Clearly, the ideal $J(f)=I\left(f_{1}, \ldots, f_{p}\right)$ does not depend on the choice of $f_{1}, \ldots, f_{p}$.

Received by the editors October 28, 1985.

1980 Mathematics Subject Classification (1985 Revision). Primary 57D45, 32B05, 14B05.

C1986 American Mathematical Society $0002-9939 / 86 \$ 1.00+\$ .25$ per page 
For $k=1, \ldots, n$, we consider $F_{k}=\mathbf{K}\left[\left[x_{1}, \ldots, x_{k}\right]\right]$ and $O_{k}=\mathbf{K}\left\{x_{1}, \ldots, x_{k}\right\}$ as subrings of $F_{n}$ and $O_{n}$, respectively.

THEOREM 1.1. Let $f$ be in $m\left(A_{n}\right)$. If the height of the ideal $J(f)$ is equal to $n-k$, then $f$ is equivalent to a polynomial in $x_{k+1}, \ldots, x_{n}$ with coefficients in $A_{k}$.

To obtain interesting corollaries we note the following.

LEMMA 1.2. For any element $f$ in $m\left(A_{n}\right)$ the height of the ideal $J(f)$ is greater than or equal to 2.

Proof. Let $f_{1}, \ldots, f_{p}$ be irreducible, relatively prime factors of $f$ and let $g=f_{1}$ $\cdots f_{p}$. One checks easily that $J(f)=I\left(f_{1}, \ldots, f_{p}\right)$ contains the ideal $I(g)$. By linearly changing variables, if necessary, and applying the Weierstrass preparation theorem, we may assume that $g=u P$, where $u$ is a unit in $A_{n}$ and $P$ is a distinguished polynomial in $x_{n}$ with coefficients in $A_{n-1}$. Clearly, $I(g)=I(P)$ and since $P$ and $\partial P / \partial x_{n}$ have no common factor, the height of $I(P)$ is greater than or equal to 2 .

Corollary 1.3. Every power series in $A_{n}, n \geqslant 2$, is equivalent to a polynomial in $x_{n-1}, x_{n}$ with coefficients in $A_{n-2}$. In particular, every element in $A_{2}$ is equivalent to a polynomial in $\mathbf{K}\left[x_{1}, x_{2}\right]$.

For arbitrary power series, Corollary 1.3 is the best result that one can get.

EXAMPLE 1.4 ([8, p. 220]). Let $\mathbf{K}=\mathbf{R}$ or $\mathbf{C}$. Then the power series $f$ in $\mathbf{K}\left\{x_{1}, x_{2}, x_{3}\right\}$ defined by

$f=x_{3}\left(x_{1}^{2}+x_{2}^{2}+2 x_{3}\right)\left(x_{1}^{2}+x_{2}^{2}+x_{3}\right)\left(x_{1}^{2}+x_{2}^{2}-\left(1+x_{1}\right) x_{3}\right)\left(x_{1}^{2}+x_{2}^{2}-e^{x_{1}} x_{3}\right)$

is not equivalent to a polynomial in $\mathbf{K}\left[x_{1}, x_{2}, x_{3}\right]$. Note that if $\mathbf{K}=\mathbf{R}$, then 0 is an isolated critical point of $f$.

If $J(f)$ has height $n$, i.e. $J(f)$ contains a certain power of the maximal ideal of $A_{n}$ or, equivalently, $A_{n} / J(f)$ is a finitely generated vector space over $\mathbf{K}$, then one can obtain a stronger result than Theorem 1.1. We say that a power series $f$ in $m\left(A_{n}\right)$ is weakly finitely determined provided that there exists a positive integer $l$ such that if $f$ is written as $f=\varepsilon f_{1}^{\lambda_{1}} \cdots f_{p}^{\lambda_{p}}$, where $\varepsilon$ belongs to $\mathbf{K}^{*}, f_{1}, \ldots, f_{p}$ are irreducible, relatively prime elements of $A_{n}$ and $\lambda_{1}, \ldots, \lambda_{p}$ are positive integers, then every power series $g$ of the form $g=\varepsilon g_{1}^{\lambda_{1}} \cdots g_{p}^{\lambda_{p}}$ with $g_{j}-f_{j}$ belonging to $m\left(A_{n}\right)^{l+1}$ for $j=1, \ldots, p$ is equivalent to $f$. In particular, every weakly finitely determined power series is equivalent to a polynomial over $\mathbf{K}$.

THEOREM 1.5. Let $f$ be in $m\left(A_{n}\right)$. If $A_{n} / J(f)$ is a finitely generated vector space over $\mathbf{K}$, then $f$ is weakly finitely determined.

We should mention a geometric interpretation of the ideal $J(f)$.

Let $\mathbf{K}$ be one of the fields $\mathbf{R}$ or $\mathbf{C}$. We shall identify elements of $O_{n}$ with germs of analytic functions at the origin in $\mathbf{K}^{n}$. Clearly, two elements $f$ and $g$ in $O_{n}$ are equivalent if and only if there exists an analytic local diffeomorphism $\sigma:\left(\mathbf{K}^{n}, 0\right) \rightarrow$ $\left(\mathbf{K}^{n}, 0\right)$ such that $g=f \circ \sigma$. Given an ideal $I$ or an element $f$ of $O_{n}, V(I)$ and $V(f)$ 
will denote the set-germs at the origin of zeros of $I$ and $f$, respectively. Let $V$ be an analytic set-germ at the origin in $\mathbf{K}^{n}$ of pure codimension one and let $V_{1}, \ldots, V_{p}$ be its irreducible analytic components. Let $W_{j}$ be a representative of $V_{j}$. Denote by $S(V)$ the germ at the origin of the set of points $x$ in $W=W_{1} \cup \cdots \cup W_{p}$ such that $W$ is not a normal crossing at $x$ or $x$ is a singular point of $W_{j}$ for some $j=1, \ldots, p$. Let $h_{j}$ be a generator of the ideal of $O_{n}$ of power series vanishing on $V_{j}$. Note that the ideal $J(V)=I\left(h_{1}, \ldots, h_{p}\right)$ does not depend on the choice of generators. Now let $f:\left(\mathbf{K}^{n}, 0\right) \rightarrow(\mathbf{K}, 0)$ be a germ of an analytic function. Write $f=\varepsilon f_{1}^{\lambda_{1}} \cdots f_{p}^{\lambda_{p}}$, where $\varepsilon$ belongs to $\mathbf{K}^{*}, f_{1}, \ldots, f_{p}$ are irreducible, relatively prime analytic germs and $\lambda_{1}, \ldots, \lambda_{p}$ are positive integers. Pick a neighborhood $U$ of the origin in $\mathbf{K}^{n}$ and a representative $g_{j}: U \rightarrow \mathbf{K}$ of $f_{j}$ for $j=1, \ldots, p$. Denote by $S(f)$ the germ at the origin of the set of points $x$ in $U$ such that the function $g=\varepsilon g_{1}^{\lambda_{1}} \cdots g_{p}^{\lambda_{p}}$ is not a normal crossing at $x$ or $g_{j}(x)=0$ and $g_{j}$ has a critical point at $x$ for some $j=1, \ldots, p$. We recall that an analtyic subset $W$ of $\mathbf{K}^{n}$ is said to be a normal crossing at a point $x$ in $W$ if its germ at $x$ is analytically isomorhic to the germ at the origin of the set $\left\{\left(y_{1}, \ldots, y_{n}\right) \in \mathbf{K}^{n} \mid y_{1} \cdots y_{i}=0\right\}$ for some $i=1, \ldots, n$. Similarly, an analytic function $g: U \rightarrow \mathbf{K}$, defined on an open set $U$ of $\mathbf{K}^{n}$, is called a normal crossing at a point $x$ in $U$ if there exists a local analytic coordinate system $\left(U_{x}, y_{1}, \ldots, y_{n}\right)$ with origin $x$, a nonvanishing analytic function $u: U_{x} \rightarrow \mathbf{K}$ and nonnegative integers $k_{1}, \ldots, k_{n}$ such that $g=u y_{1}^{k_{1}} \cdots y_{n}^{k_{n}}$ on $U_{x}$.

Proposition 1.6. Let $\mathbf{K}$ be one of the fields $\mathbf{R}$ or $\mathbf{C}$. For any $f$ in $m\left(O_{n}\right)$ one has $V(J(f))=S(f)$. Moreover, if $\mathbf{K}=\mathbf{C}$, then $J(f)=J(V(f)), V(J(f))=S(V(f))$ and the height of $J(f)$ is equal to the codimension of $S(V(f))$.

Proof. An exercise. The last part follows from the Nullstellensatz.

As we saw, in the complex case, the ideal $J(f)$ is completely determined by $V(f)$. From Theorem 1.1, one obtains immediately:

Corollary 1.7. Let $f$ be in $\mathbf{C}\left\{x_{1}, \ldots, x_{n}\right\}$ and $f(0)=0$. If $\operatorname{codim} S(V(f))=n-$ $k$, then $f$ is equivalent to a polynomial in $x_{k+1}, \ldots, x_{n}$ with coefficients in $\mathbf{C}\left\{x_{1}, \ldots, x_{k}\right\}$.

Over the field $\mathbf{R}$ information about $V(f)$ is, usually, insufficient to draw some conclusions about $f$. Indeed, $f$ can have factors vanishing only at the origin, $V(f)$ need not be coherent, etc. Clearly, $S(V(f)) \subseteq S(f)$ but, in general, these two germs are different. Moreover, the height of $J(f)$ is less than or equal to the codimension of $V(J(f))=S(f)$ but the strong inequality can occur. For instance, taking $f$ of Example 1.4, one has $S(f)=\{0\}$ but $f$ is not equivalent to a polynomial over $\mathbf{R}$ and thus, according to Theorem 1.1, the height of $J(f)$ is less than 3. Nevertheless, we have the following.

THEOREM 1.8. Let $f$ be in $\mathbf{R}\left\{x_{1}, \ldots, x_{n}\right\}, f(0)=0$, and let $r$ be a nonnegative integer. If the codimension of $S(f)$ is equal to $n-k$, then there exists a local $C^{r}$ diffeomorphism $\sigma:\left(\mathbf{R}^{n}, 0\right) \rightarrow\left(\mathbf{R}^{n}, 0\right)$ such that $f \circ \sigma$ is a polynomial in $x_{k+1}, \ldots, x_{n}$ with coefficients in $\mathbf{R}\left\{x_{1}, \ldots, x_{k}\right\}$. 
It follows from Example 1.4 that $\sigma$ cannot be replaced by an analytic diffeomorphism.

Our method also works for $C^{\infty}$ functions. Let $E_{n}$ be the ring of germs at the origin of $C^{\infty}$ functions on $\mathbf{R}^{n}$ and let $T$ be the homomorphism from $E_{n}$ onto $\mathbf{R}\left[\left[x_{1}, \ldots, x_{n}\right]\right]$ induced by the Taylor expansion at the origin. We say that an element $f$ in $E_{n}$ is factorizable if it can be written as $f=\varepsilon f_{1}^{\lambda_{1}} \cdots f_{p}^{\lambda_{p}}$, where $\varepsilon$ is in $\mathbf{R}^{*}, \lambda_{1}, \ldots, \lambda_{p}$ are positive integers, $f_{1}, \ldots, f_{p}$ belong to $E_{n}$ and $T f_{1}, \ldots, T f_{p}$ are irreducible, relatively prime elements of $\mathbf{R}\left[\left[x_{1}, \ldots, x_{n}\right]\right]$. Two function-germs $f$ and $g$ in $E_{n}$ are said to be equivalent if there exists a local $C^{\infty}$ diffeomorphism $\sigma$ : $\left(\mathbf{R}^{n}, 0\right) \rightarrow\left(\mathbf{R}^{n}, 0\right)$ such that $g=f \circ \sigma$.

THEOREM 1.9. Let $f$ be a factorizable element of $E_{n}$. If the height of the ideal $J(T f)$ is equal to $n-k$, then $f$ is equivalent to a polynomial in $x_{k+1}, \ldots, x_{n}$ with coefficients in $E_{k}$.

From Theorem 1.9 and Lemma 1.2 one obtains immediately

COROLlaRY 1.10. Every factorizable element in $E_{n}$ is equivalent to a polynomial in $x_{n-1}, x_{n}$ with coefficients in $E_{n-2}$. In particular, an element $f$ of $E_{2}$ is equivalent to a polynomial in $\mathbf{R}\left[x_{1}, x_{2}\right]$ if and only if $f-f(0)$ is factorizable.

To trace the history of the problem the reader may consult $[4,5,8,10]$. The main results, Theorems 1.1, 1.8, 1.9 and Corollary 1.7 , are new and considerably stronger than the ones previously known. Corollary 1.3 , for $\mathbf{K}=\mathbf{R}$ and for convergent power series over $\mathbf{K}=\mathbf{C}$ as well as Corollary 1.10 have been proved in [7]. The ideal $J(f)$ has been introduced in the paper [2], which also contains a proof of Theorem 1.5 for convergent power series over $\mathbf{R}$ or $\mathbf{C}$. The second part of Corollary 1.3 and a different version of Theorem 1.5, both for convergent power series over $\mathbf{C}$, are in [3]. It should be mentioned that the proofs in [2,3, and 7] do not carry over on power series over an arbitrary field of characteristic 0 (not even on formal power series over C). Moreover, what perhaps is more interesting, the proofs presented in this note are simpler even for $\mathbf{K}=\mathbf{R}$ or $\mathbf{C}$.

2. Some ideals associated with power series. Let $\varphi=\left(\varphi_{1}, \ldots, \varphi_{p}\right)$ be a $p$-tuple of elements of $A_{n}$ and let $\lambda=\left(\lambda_{1}, \ldots, \lambda_{p}\right)$ be a $p$-tuple of positive integers. We denote by $I(\varphi ; \lambda)$ the ideal of $A_{n}$ generated by all $(p+1) \times(p+1)$ minors of the $(p+1) \times(p+n)$ matrix

$$
M(\varphi ; \lambda)=\left(\begin{array}{ccccccc}
\varphi_{1} & 0 & \cdots & 0 & \frac{\partial \varphi_{1}}{\partial x_{1}} & \cdots & \frac{\partial \varphi_{1}}{\partial x_{n}} \\
0 & \varphi_{2} & \cdots & 0 & \frac{\partial \varphi_{2}}{\partial{ }_{1}} & \cdots & \frac{\partial \varphi_{2}}{\partial x_{n}} \\
\vdots & \vdots & & \vdots & \vdots & & \vdots \\
0 & 0 & \cdots & \varphi_{p} & \frac{\partial \varphi_{p}}{\partial x_{1}} & \cdots & \frac{\partial \varphi_{p}}{\partial x_{n}} \\
\lambda_{1} & \lambda_{2} & \cdots & \lambda_{p} & 0 & \cdots & 0
\end{array}\right) .
$$

The following observation will play an important role. 
LeMma 2.1. Let $\varphi=\left(\varphi_{1}, \ldots, \varphi_{p}\right)$ be a p-tuple of elements of $m\left(A_{n}\right)$ and let $\lambda=\left(\lambda_{1}, \ldots, \lambda_{p}\right)$ be a p-tuple of positive integers. Then $I(\varphi ; \lambda)$ is contained in $I(\varphi)$ and the radicals of both ideals are equal.

Proof. Since the ring $F_{n}$ is flat over $O_{n}$, it suffices to prove the lemma for $A_{n}=F_{n}$. The first part of the conclusion is obvious. To show the second part we may assume that $\mathbf{K}$ is an algebraically closed field. Indeed, the general case follows easily by passing to the ring of formal power series over the algebraic closure of $\mathbf{K}$. By [10, p. 49], we only have to prove that if $H: F_{n} \rightarrow \mathbf{K}[[t]]$ is a $\mathbf{K}$-homomorphism of algebras vanishing on $I(\varphi ; \lambda)$, then it also vanishes on $I(\varphi)$. Let $f=\varphi_{1}^{\lambda_{1}} \cdots \varphi_{p}^{\lambda_{p}}$. Note that for each $i=1, \ldots, n$,

$$
\frac{\partial f}{\partial x_{i}}=\varphi_{1}^{\lambda_{1}-1} \cdots \varphi_{p}^{\lambda_{p}-1}\left(\lambda_{1} \frac{\partial \varphi_{1}}{\partial x_{i}} \varphi_{2} \cdots \varphi_{p}+\cdots+\lambda_{p} \varphi_{1} \cdots \varphi_{p-1} \frac{\partial \varphi_{p}}{\partial x_{i}}\right)
$$

belongs to $I(\varphi ; \lambda)$ and hence, by $[10$, p. 50], $H(f)=0$. Clearly, the rows of the matrix $M(\varphi ; \lambda)_{H}$ obtained by applying $H$ to each entry in $M(\varphi ; \lambda)$ are linearly dependent over the field $\mathbf{L}$ of fractions of $\mathbf{K}[[t]]$. Since $H\left(\varphi_{j}\right)=0$ for some $j=1, \ldots, p$, the last row $\left(\lambda_{1}, \lambda_{2}, \ldots, \lambda_{p}, 0, \ldots, 0\right)$ is not a linear combination of the first $p$ rows of $M(\varphi ; \lambda)_{H}$. Thus the first $p$ rows of $M(\varphi ; \lambda)_{H}$, which are the same as the rows of the matrix obtained by applying $H$ to each entry in $M(\varphi)$, are linearly dependent over $\mathbf{L}$ and $H$ vanishes on $I(\varphi)$.

REMARK 2.2. For convergent power series over $\mathbf{C}$ one can also prove Lemma 2.1 by applying the classical Nullstellensatz.

3. The proofs. We only have to prove Theorems $1.1,1.5,1.8$, and 1.9.

Proof of Theorem 1.1. Let $f=\varepsilon f_{1}^{\lambda_{1}} \cdots f_{p}^{\lambda_{p}}$, where $\varepsilon$ is in $\mathbf{K}^{*}, f_{1}, \ldots, f_{p}$ are irreducible, relatively prime elements in $A_{n}$ and $\lambda_{1}, \ldots, \lambda_{p}$ are positive integers. Let $\varphi=\left(f_{1}, \ldots, f_{p}\right)$ and $\lambda=\left(\lambda_{1}, \ldots, \lambda_{p}\right)$. By Lemma 2.1 , the height of the ideal $I=m\left(A_{n}\right)^{2} I(\varphi ; \lambda)^{2}$ is equal to $n-k$. Now, linearly changing variables and applying the normalization theorem for power series, we may assume that the ideal generated by $x_{1}, \ldots, x_{k}$ and $I$ contains a certain power of $m\left(A_{n}\right)$. Hence $I+$ $O_{k}\left[x_{k+1}, \ldots, x_{n}\right]=O_{n}$. In particular, one can find elements $g_{1}, \ldots, g_{p}$ in $O_{k}\left[x_{k+1}, \ldots, x_{n}\right]$ such that $f_{j}-g_{j}$ belongs to $I$ for $j=1, \ldots, p$. Now set $x=$ $\left(x_{1}, \ldots, x_{n}\right), y=\left(y_{1}, \ldots, y_{p}\right), z=\left(z_{1}, \ldots, z_{n}\right)$ and consider $F=\left(F_{1}, \ldots, F_{p}, F_{p+1}\right)$ defined by

$$
F_{j}(x, y, z)=\left(1+y_{j}\right) f_{j}(x+z)-g_{j}(x)
$$

for $j=1, \ldots, p$ and

$$
F_{p+1}(x, y, z)=\left(1+y_{1}\right)^{\lambda_{1}} \cdots\left(1+y_{p}\right)^{\lambda_{p}}-1 .
$$

The Jacobian matrix $F_{(y, z)}^{\prime}(x, 0,0)$ is equal to $M(\varphi ; \lambda)$. By Tougeron's implicit function theorem [10, p. 57], there exist power series $y(x)=\left(y_{1}(x), \ldots, y_{p}(x)\right)$ and $z(x)=\left(z_{1}(x), \ldots, z_{n}(x)\right)$ such that $F(x, y(x), z(x))=0$ and $y_{j}(x), z_{i}(x)$ belong to $m\left(A_{n}\right)^{2}$ for $j=1, \ldots, p, i=1, \ldots, n$. Now $\sigma(f)=g$, where $g=\varepsilon g_{1}^{\lambda_{1}} \cdots g_{p}^{\lambda_{p}}$ and $\sigma$ is the K-automorphism of $A_{n}$ defined by $\sigma(h(x))=h(x+z(x))$ for $h(x)$ in $A_{n}$. 
Proof OF Theorem 1.5. Let $f=\varepsilon f_{1}^{\lambda_{1}} \cdots f_{p}^{\lambda_{p}}$, where $\varepsilon$ belongs to $\mathbf{K}^{*}, f_{1}, \ldots, f_{p}$ are irreducible, relatively prime elements of $A_{n}$ and $\lambda_{1}, \ldots, \lambda_{p}$ are positive integers. Thanks to Lemma 2.1 , one can find a positive integer $l$ such that $m\left(A_{n}\right)^{l+1}$ is contained in $m\left(A_{n}\right)^{2} I(\varphi ; \lambda)^{2}$, with $\varphi=\left(f_{1}, \ldots, f_{p}\right)$ and $\lambda=\left(\lambda_{1}, \ldots, \lambda_{p}\right)$. Let $g_{1}, \ldots, g_{p}$ be elements of $A_{n}$ such that $g_{j}-f_{j}$ belongs to $m\left(A_{n}\right)^{l+1}$ for $j=1, \ldots, p$. Now, by considering $F=\left(F_{1}, \ldots, F_{p}, F_{p+1}\right)$ defined as in the proof of Theorem 1.1, the conclusion follows.

Proof OF Theorem 1.9. Let $f=\varepsilon f_{1}^{\lambda_{1}} \cdots f_{p}^{\lambda_{p}}$, where $\varepsilon$ is in $\mathbf{R}^{*}, \lambda_{1}, \ldots, \lambda_{p}$ are positive integers, $f_{1}, \ldots, f_{p}$ belong to $E_{n}$ and $T f_{1}, \ldots, T f_{p}$ are irreducible relatively prime elements of $\mathbf{R}\left[\left[x_{1}, \ldots, x_{n}\right]\right]$. Let $\varphi=\left(f_{1}, \ldots, f_{p}\right), \lambda=\left(\lambda_{1}, \ldots, \lambda_{p}\right)$ and let $I(\varphi ; \lambda)$ be the ideal of $E_{n}$ defined in the same way as the corresponding ideal in the case of power series. Denote by $m\left(E_{n}\right)$ the maximal ideal of $E_{n}$. By Lemma 2.1, the ideal $T(I)$ of $\mathbf{R}\left[\left[x_{1}, \ldots, x_{n}\right]\right]$, where $I=m\left(E_{n}\right)^{2} I(\varphi ; \lambda)^{2}$, has height $n-k$. Thanks to $[10$, p. 168], we may assume that the coordinates have been chosen such that there exist $g_{1}, \ldots, g_{p}$ which are polynomials in $x_{k+1}, \ldots, x_{n}$ with coefficients in $E_{k}$ and $f_{j}-g_{j}$ belongs to $I$ for $j=1, \ldots, p$. Let $F=\left(F_{1}, \ldots, F_{p}, F_{p+1}\right)$ be defined as in the proof of Theorem 1.1. The conclusion follows by applying Tougeron's implicit function theorem for $C^{\infty}$ functions [8].

In the proof of Theorem 1.8 it will be convenient to identify set-germs and function-germs with their representatives.

Proof OF TheOrem 1.8. Write $f=\varepsilon f_{1}^{\lambda_{1}} \cdots f_{p}^{\lambda_{p}}$, where $\varepsilon$ is in $\mathbf{R}^{*}, f_{1}, \ldots, f_{p}$ are irreducible, relatively prime elements of $O_{n}$ and $\lambda_{1}, \ldots, \lambda_{p}$ are positive integers. Let $\varphi=\left(f_{1}, \ldots, f_{p}\right)$ and $\lambda=\left(\lambda_{1}, \ldots, \lambda_{p}\right)$. Choose an element $h$ in the ideal $I=$ $m\left(O_{n}\right)^{2} I(\varphi ; \lambda)^{2}$ such that $h \geqslant 0$ and $V(h)=V(I)$. By the Lojasiewicz inequality [10], there exist positive real numbers $c, \eta$ and $\alpha$ such that

$$
h(x) \geqslant c d(x, V(h))^{\alpha} \quad \text { for }\|x\|<\eta,
$$

where $d(x, V(h))$ denotes the distance from $x$ to $V(h)$. Now take a positive integer $l$ such that for each analytic function $u$ defined in a neighborhood of 0 in $\mathbf{R}^{n}$ and $l$-flat on $V(h)$, the function $u / h$ extends to a $C^{r+3}$ function on a neighborhood of 0 vanishing on $V(h)$. Let $J$ be the ideal of elements of $O_{n}$ vanishing on $S(f)$. Clearly, $J$ as well as $J^{l+1}$ have height $n-k$. By Proposition 1.6 and Lemma 2.1, $V(h)=$ $S(f)$. Thanks to the normalization theorem, we may assume that $J^{l+1}+$ $O_{k}\left[x_{k+1}, \ldots, x_{n}\right]=O_{n}$. Denote by $E_{n}^{(r+3)}$ the ring of germs at the origin of $C^{r+3}$ functions on $\mathbf{R}^{n}$. Note that $J^{I+1}$ is contained in $I E_{n}^{(r+3)}$. Thus one can find elements $g_{1}, \ldots, g_{p}$ in $O_{k}\left[x_{k+1}, \ldots, x_{n}\right]$ such that $f_{j}-g_{j}$ belongs to $I E_{n}^{(r+3)}$ for $j=1, \ldots, p$. Now consider $F=\left(F_{1}, \ldots, F_{p}, F_{p+1}\right)$ defined as in the proof of Theorem 1.1. By applying Tougeron's implicit function theorem for finitely differentiable functions $[1,9]$, one concludes the proof.

\section{REFERENCES}

1. J. Bochnak, Relèvement de jets, Séminaire Pierre Lelong (Analyse) Année 1970-1971, Lecture Notes in Math., vol. 275, Springer, pp. 106-118.

2. J. Bochnak, W. Kucharz, and M. Shiota, On algebraicity of global real analytic sets and functions, Invent. Math. 70 (1982). 115-156.

3. D. Cerveau and J. F. Mattei, Formes intégrables holomorphes singulières, Astérisque 97 (1982). 
4. N. Levinson, A canonical form for an analytic function of several variables at a critical point, Bull. Amer. Math. Soc. 66 (1960), 68-69.

5. A polynomial canonical form for certain analytic functions at two variables at a critical point, Bull. Amer. Math. Soc. 68 (1960), 366-368.

6. M. Nagata, Local rings, Wiley, 1962.

7. M. Shiota, Equivalence of differentiable mappings and analytic mappings, Inst. Hautes Études Sci. Publ.Math. 54 (1981), 37-122.

8. J. A. Tougeron, Idéaux de fonctions differentiables. I, Ann. Inst. Fourier (Grenoble) 18 (1968), 177-240.

9. , G-stabilité des germes d'applications différentiables, preprint, Université de Rennes, 1971.

10. Idéaux de fonctions différentiables, Ergeb. Math. Grenzgeb., Band 71, Springer-Verlag, Berlin and New York, 1972.

Department of Mathematics and Statistics, University of New Mexico, Albuquerque, New MeXICO 87131 